Commun. math. Phys. 21, 150-163 (1971)

(C) by Springer-Verlag 1971

\title{
Linear Kinematical Groups
}

\author{
VITTORIO GORINI ${ }^{\star}$ \\ Institut für Theoretische Physik (I) der Universität Marburg, Germany
}

Received November 12, 1970

\begin{abstract}
We prove a theorem which states that in an $(n+1)$-dimensional space-time $(n \geqq 3)$ the only linear kinematical groups which are compatible with the isotropy of space are the Lorentz and Galilei groups. The special cases $n=1$ and $n=2$ are also briefly discussed.
\end{abstract}

\section{Introduction}

We prove in this paper that in an $(n+1)$-dimensional space-time $(n \geqq 3)$ the only non trivial linear kinematical groups which are compatible with the isotropy of space are the Lorentz and Galilei groups.

Related to ours are the papers by Lalan [1] and by Bacry and LévyLeblond [2]. Lalan's conditions are however much more restrictive than ours in that he assumes at the outset a Lie group structure and he requires the set of special velocity transformations to be invariant under space rotations. As to the approach of Ref. [2], it is more general than ours because space-time translations are considered as well and no restriction to linearity is introduced. On the other hand, the Lie group assumption is still used and, furthermore, invariance under parity and time reversal is required.

In Section 2 we collect some notations. In Section 3 we discuss our assumptions. In Section 4 we state and prove our theorem and mention its extension to the inclusion of space reflection, as a corollary ${ }^{1}$. In Section 5 the cases $n=1$ and $n=2$ are discussed. For $n=1$, some specific counterexamples are listed, which prove that the result no longer holds. As for the case $n=2$, it is seen to hold if space reflection is allowed for, or under restriction to connected Lie groups.

\section{Notations}

Let $m$ and $n$ be positive integers. We use the standard notations $\mathrm{GL}(n+1, R)$ for the group of all $(n+1) \times(n+1)$ real non singular matrices

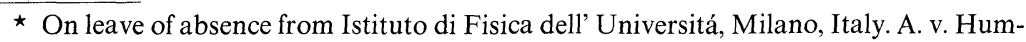
boldt fellow.

${ }^{1}$ For the case $n=3$ the result was communicated in [3]. Compare also Ref. [4] where, however, an unnecessary strong continuity condition was used. 
and $O(m)$ (respectively, $S O(m)$ ) for the group of all $m \times m$ real orthogonal matrices (respectively, orthogonal matrices with determinant plus one). Greek indices run from 1 to $n+1$, latin indices from 1 to $n$. A matrix $G$ belonging to $\operatorname{GL}(n+1, R)$ will often be denoted by the family of its matrix elements $G=\left\{G_{\mu v}\right\}_{\mu, v=1, \ldots, n+1}=\left\{G_{\mu v}\right\}$. We shall have to consider the following subgroups of $\mathrm{GL}(n+1, R)$ :

$$
\begin{aligned}
\mathscr{H}(n)= & \left\{H \mid H \in \mathrm{GL}(n+1, R) ; H_{i, n+1}=0, i=1, \ldots, n\right\}, \\
\overline{\mathscr{C}}(n)= & \left\{C \mid C \in \mathrm{GL}(n+1, R) ; C_{i, n+1}=C_{n+1, i}=0,\right. \\
& \left.i=1, \ldots, n ; C_{n+1, n+1}=1 ;\left\{C_{i k}\right\} \in O(n)\right\}, \\
\mathscr{C}(n)= & \left\{C \mid C \in \overline{\mathscr{C}}(n), \operatorname{det}\left\{C_{i k}\right\}=1\right\}, \\
\mathscr{C}^{\prime}(n)= & \left\{C \mid C \in \mathscr{C}(n), C_{11}=1\right\}, \\
\overline{\mathscr{L}}(n, \lambda)= & \left\{L \mid L \in \mathrm{GL}(n+1, R) ; L^{T} g(\lambda) L=g(\lambda) ;\right. \\
& g_{\mu \nu}(\lambda)=0 \text { if } \quad \mu \neq v ; g_{11}(\lambda)=\cdots=g_{n n}(\lambda)=-\lambda ; \\
& \left.g_{n+1, n+1}(\lambda)=1 ; L_{n+1, n+1} \geqq 1\right\}, \\
& 0<\lambda<+\infty, \\
\mathscr{L}(n, \lambda)= & \{L \mid L \in \overline{\mathscr{L}}(n, \lambda) ; \operatorname{det} L=1\}, \quad 0<\lambda<+\infty, \\
\overline{\mathscr{G}}(n)= & \left\{G \mid G \in \mathrm{GL}(n+1, R) ; G_{n+1, i}=0, i=1, \ldots, n ;\right. \\
& \left.G_{n+1, n+1}=1 ;\left\{G_{j k}\right\} \in O(n)\right\}, \\
\mathscr{G}(n)= & \{G \mid G \in \overline{\mathscr{G}}(n) ; \operatorname{det} G=1\}
\end{aligned}
$$

and, for $n \geqq 2$,

$$
\begin{aligned}
\mathscr{X}(n)= & \left\{X \mid X \in \mathrm{GL}(n+1, R) ; X_{l 1}=X_{l, n+1}=0,\right. \\
& l=2, \ldots, n ; \Delta(X)=X_{11} X_{n+1, n+1} \\
& \left.-X_{1, n+1} X_{n+1,1}>0\right\} .
\end{aligned}
$$

If $C \in \mathscr{C}(n)$, we write $C=C^{(i, k)}(\alpha), i<k, \alpha \in[0,2 \pi)$, if $C_{j j}=1$ for $j \neq i, k$ and $C_{i i}=C_{k k}=\cos \alpha, C_{i k}=-C_{k i}=\sin \alpha$.

\section{Discussion of the Assumptions}

Let $M=\left\{\boldsymbol{x}=\left(x_{1}, \ldots, x_{n}\right), t=x_{n+1}\right\}_{\boldsymbol{x} \in R^{n}, t \in R}$ be an $(n+1)$-dimensional space-time (without metric) ${ }^{2}$. We restrict ourselves to (inertial) frames having the same space-time origin and we assume linearity. Hence we

\footnotetext{
${ }^{2}$ We assume $R^{n}$ to be equipped with the usual euclidean metric.
} 
write the coordinate transformation between two frames $S$ and $S^{\prime}$ as

$$
x_{\mu}^{\prime}=\sum_{v=1}^{n+1} L_{\mu \nu} x_{v},
$$

where $\left\{L_{\mu \nu}\right\} \in \mathrm{GL}(n+1, R)$, and denote by $\mathscr{L}$ the group of such transformations, identified to the group of the corresponding matrices $\left\{L_{\mu \nu}\right\}$ 's. Let $\mathscr{L}_{R}$ denote the subgroup of $\mathscr{L}$ whose elements are the transformations connecting frames at rest with respect to each other. The condition for $S^{\prime}$ to be at rest with respect to $S$ is expressed by the requirement that a world line which is parallel to the $x_{n+1}$-axis should be transformed by (3.1) into a world line which is parallel to the $x_{n+1}^{\prime}$-axis. This is equivalent to

and therefore

$$
L_{i, n+1}=0, \quad i=1, \ldots, n
$$

$$
\mathscr{L}_{R}=\mathscr{L} \cap \mathscr{H}(n) .
$$

\section{Axiom}

$$
\mathscr{L}_{R}=\mathscr{C}(n) .
$$

Physically, this axiom states that a) in each frame clocks have been synchronized in a standard way (here space-time homogeneity and isotropy of space are implicit), b) that time has a unidirectional flow and that a common time standard is used, c) that space is isotropic and that the localization of events in space is given by orthogonal coordinate systems of the same parity, a common length standard being adopted.

By (3.3) and (3.4) the kinematical group must satisfy

$$
\mathscr{L} \cap \mathscr{H}(n)=\mathscr{C}(n) .
$$

We prove in the next section that, if $n \geqq 3$, the subgroups $\mathscr{L}$ of $\mathrm{GL}(n+1, R)$ satisfying (3.6) are, apart from $\mathscr{C}(n)$ itself, the proper orthochronous Galilei group (2.8) and the proper orthochronous Lorentz groups (2.6) corresponding to all possible real values of the invariant velocity $\lambda^{-1 / 2}$.

\section{The Theorem}

Theorem 1. Let $n \geqq 3$ and denote by $\Phi$ the family of subgroups of $\mathrm{GL}(n+1, R)$ defined by $\mathscr{L} \in \Phi$ iff $\mathscr{L} \cap \mathscr{H}(n)=\mathscr{C}(n)$. Then $\Phi=\left\{\mathscr{G}_{\lambda}\right\}_{\lambda \in[0,+\infty]^{\prime}}$ where a) if $\left.\left.0<\lambda<+\infty, \mathscr{G}_{\lambda}=\mathscr{L}(n, \lambda) ; b\right) \mathscr{G}_{0}=\mathscr{G}(n) ; c\right) \mathscr{G}_{+\infty}=\mathscr{C}(n)$.

Lemma 1. Let $\mathscr{L} \in \Phi$ and let $L \in \mathscr{L}$. Then $L$ can be written in the form

$$
L=A X B,
$$

where $A, B \in \mathscr{C}(n), X \in \mathscr{X}(n)$ and

$$
X_{11} \neq 0, X_{n+1, n+1}=L_{n+1, n+1} \neq 0 .
$$


Proof. By the properties of $S O(n)$ it is a trivial matter to verify that two matrices $C, C^{\prime} \in \mathscr{C}(n)$ can be found such that $\left(C L C^{\prime}\right)_{l 1}=\left(C L C^{\prime}\right)_{l, n+1}=0$, $l=2, \ldots, n$. Since det $Y \neq 0$ we have $\Delta(Y) \neq 0$. Therefore we can take $X=\left[C^{(1,2)}(\pi)\right]^{m} Y, A=C^{-1}\left[C^{(1,2)}(\pi)\right]^{m}, B=C^{\prime-1}$ with $m=0$ or $m=1$ according to whether $\Delta(Y)>0$ or $\Delta(Y)<0$. One then controls that $X_{11}=0$ (respectively, $\left.X_{n+1, n+1}=0\right)$ implies $\left(X C^{(1,2)}(\pi) X^{-1}\right)_{\mu, n+1}$ $=-\delta_{\mu, n+1}\left(\right.$ respectively, $\left.\left(X^{-1} C^{(1,2)}(\pi) X\right)_{\mu, n+1}=-\delta_{\mu, n+1}\right)$ and this contradicts (3.5).

Lemma 2. Let $D \in \operatorname{GL}(m, R)$ with the property that $\forall F \in S O(m)$ $\exists F^{\prime} \in S O(m)$ such that

$$
D F=F^{\prime} D .
$$

Then $D=b Q, b>0, Q \in O(m)$.

Proof. The statement is trivial for $m=1$, so we suppose $m \geqq 2$. We have $I=\left(D F D^{-1}\right)^{T}\left(D F D^{-1}\right)=\left(D^{T}\right)^{-1} F^{T}\left(D^{T} D\right) F D^{-1}$. Hence the positive symmetric matrix $E=D^{T} D$ commutes with every rotation

$$
F E=E F, \forall F \in S O(m) \text {. }
$$

$\exists N \in O(m)$ such that $N E N^{-1}$ is diagonal [5]:

$$
N E N^{-1}=\tilde{E}=\operatorname{diag}\left\{a_{1}, \ldots, a_{n}\right\}
$$

and $E$ commutes with every rotation as well. Then, denoting by $F^{(i, j)}(\alpha)$ $(1 \leqq i<j \leqq m)$ a rotation of $\alpha$ in the $(i, j)$-plane, from $\tilde{E} F^{(i, j)}(\pi / 2)=F^{(i, j)}(\pi / 2) \tilde{E}$ we get $a_{i}=a_{j}$, whence $\tilde{E}=a I=E=D^{T} D, a>0$. Therefore we get the result with $b=a^{1 / 2}$.

Now let $\mathscr{L} \in \Phi$ and define

and $\forall v \in \mathscr{V}$,

$$
\begin{gathered}
\mathscr{K}=\mathscr{L} \cap \mathscr{X}(n), \\
\mathscr{V}=\left\{v \mid v=-X_{1, n+1} / X_{11}, X \in \mathscr{K}\right\}
\end{gathered}
$$

Note that, by Lemma $1, \mathscr{V}$ is a subset of the real line $R$.

Lemma 3. There exists an injection $N: v \rightarrow N(v)$ of $\mathscr{V}$ into $\mathrm{GL}(n+1, R)$ such that, $\forall v \in \mathscr{V}$,

$$
\mathscr{K}(v)=\left\{K \mid K=N(v) C ; C \in \mathscr{C}^{\prime}(n)\right\},
$$

and

$$
N_{\mu v}(v)=0 \quad \text { if } \quad \mu \neq v \quad \text { but } \quad(\mu, v) \neq(1, n+1)
$$

and $(\mu, v) \neq(n+1,1)$,

$$
\begin{aligned}
N_{11}(v) & =a(v), \quad N_{1, n+1}(v)=-v a(v), \\
N_{n+1,1}(v) & =c(v), \quad N_{n+1, n+1}(v)=d(v), \\
N_{22}(v) & =\cdots=N_{n-1, n-1}(v)=e(v), \quad N_{n n}(v)=f(v),
\end{aligned}
$$


with

$$
e(v)>0, \quad f^{2}(v)=e^{2}(v) .
$$

Proof. Let $v \in \mathscr{V}$ and let $X, Y \in \mathscr{K}(v)$. One checks that $\left(X Y^{-1}\right)_{i, n+1}$ $=0, i=1, \ldots, n$, hence, by (3.5), $X Y^{-1}=C \in \mathscr{C}(n)$. We have

$$
X_{i 1}=\sum_{k=1}^{n} C_{i k} Y_{k 1}=C_{i 1} Y_{11}
$$

and since $Y_{11} \neq 0$ (Lemma 1), we get $C_{21}=\cdots=C_{n 1}=0$, hence $C_{11}= \pm 1$. The circumstance $C_{11}=-1$ has to be excluded because it would imply $\Delta(X)=-\Delta(Y)$ and we conclude that $X=C Y, C \in \mathscr{C}^{\prime}(n)$. Let now $K \in \mathscr{K}(v)$ and $D \in \mathscr{C}^{\prime}(n)$. Then $K D \in \mathscr{K}(v)$ and we have from the preceding that $\exists D^{\prime} \in \mathscr{C}^{\prime}(n)$ such that

$$
K D=D^{\prime} K
$$

We have $\left(D^{\prime} K\right)_{1 i}=D_{11}^{\prime} K_{1 i}=K_{1 i}=(K D)_{1 i}=K_{11} D_{1 i}+\sum_{l=2}^{n} K_{1 l} D_{l i}$. For $i=2, \ldots, n$,

$$
K_{1 i}=\sum_{l=2}^{n} K_{1 l} D_{l i}
$$

Therefore, since the matrix $\tilde{D}=\left\{D_{l i}\right\}_{l, i=2, \ldots, n}$ is arbitrary in $S O(n-1)$ and $n \geqq 3$, we get

$$
K_{12}=\cdots=K_{1 n}=0
$$

In the same way we obtain

$$
K_{n+1,2}=\cdots=K_{n+1, n}=0 .
$$

From (4.12) we also have, for $i, j=2, \ldots, n$,

$$
\sum_{l=2}^{n} K_{i l} D_{l j}=\sum_{l=2}^{n} D_{i l}^{\prime} K_{l j}
$$

Therefore, by Lemma 2,

$$
\tilde{K}=\left\{K_{i l}\right\}_{i, l=2, \ldots, n}=e Q, e>0, Q \in O(n-1) .
$$

Now let $K_{1}$ and $K_{2}$ be elements of $\mathscr{K}(v)$. By Eqs. (4.14), (4.15), and (4.17) we can write

$$
K_{1,2}=\left(\begin{array}{ccc}
a_{1,2} & \mathbf{0} & -v a_{1,2} \\
\mathbf{0} & e_{1,2} Q_{1,2} & \mathbf{0} \\
c_{1,2} & \mathbf{0} & d_{1,2}
\end{array}\right) .
$$


There is a $C \in \mathscr{C}^{\prime}(n)$ such that

$$
K_{1}=C K_{2} .
$$

Inserting (4.18) into (4.19) we get $a_{1}=a_{2}=a, c_{1}=c_{2}=c, d_{1}=d_{2}=d$ and

$$
e_{1} Q_{1}=e_{2} \tilde{C} Q_{2},
$$

where $\tilde{C}=\left\{C_{i l}\right\}_{i, l=2, \ldots, n}$. Taking determinants in Eq. (4.21) and recalling that $e_{1}>0, e_{2}>0$, we get $e_{1}=e_{2}=e$ and $\operatorname{det} Q_{1}=\operatorname{det} Q_{2}$. Therefore, the generical element of $\mathscr{K}(v)$ can be written as

$$
K=\left(\begin{array}{ccc}
a(v) & \mathbf{0} & -v a(v) \\
\mathbf{0} & e(v) \tilde{C} Q & \mathbf{0} \\
c(v) & \mathbf{0} & d(v)
\end{array}\right)
$$

where $Q$ is a given element of $O(n-1)$ and $C$ is an arbitrary element of $S O(n-1)$. This proves the statement of the lemma with $f(v)=+e(v)$ or $f(v)=-e(v)$ according to whether $\operatorname{det} Q=1$ or $\operatorname{det} Q=-1$.

The set $\mathscr{N}=N(\mathscr{V})$ is a subgroup of $\mathscr{K}$ and, by Lemmas 1 and 3 we have

$$
\mathscr{L}=\{L \mid L=A Z B ; A, B \in \mathscr{C}(n) ; Z \in \mathscr{N}\} .
$$

Proof of the Theorem. Note first that $\mathscr{C}^{\prime}(n)=\mathscr{C}(n) \cap \mathscr{K}=\mathscr{K}(0)$. Hence $0 \in \mathscr{V}$, and if $\mathscr{V}=\{0\}$ we have $\mathscr{N}=\{N(0)\}=\{I\}$ whence, by (4.23), $\mathscr{L}=\mathscr{C}(n)=\mathscr{G}_{+\infty}$, which is the trivial solution. If $\mathscr{V} \supset\{0\}$ we fix a $v \in \mathscr{V}, v \neq 0$, and calculate

$$
\begin{aligned}
& L(\varepsilon, v)=N^{-1}(v) C^{(1,2)}(-\varepsilon) N(v) \\
& \left(\begin{array}{cccc}
\frac{d \cdot \cos \varepsilon+v c}{d+v c} & \frac{-d e \cdot \sin \varepsilon}{a(d+v c)} & 0 \ldots 0 & \frac{d v(1-\cos \varepsilon)}{d+v c} \\
\frac{a \cdot \sin \varepsilon}{e} & \cos \varepsilon & 0 \ldots 0 & \frac{-v a \cdot \sin \varepsilon}{e} \\
0 & 0 & 1 \ldots 0 & 0 \\
\ldots \ldots \ldots \ldots \ldots \ldots \ldots \ldots \ldots \ldots \ldots & 0 \\
0 & 0 & 0 \ldots \ldots \ldots \ldots \ldots \ldots & \frac{d+v c \cdot \cos \varepsilon}{d+v c}
\end{array}\right), \\
& \varepsilon \in[0,2 \pi) .
\end{aligned}
$$


By Lemma 1 (see (4.2)), we have

$$
\frac{d(v)+v c(v) \cos \varepsilon}{d(v)+v c(v)}>0, \quad \varepsilon \in[0,2 \pi),
$$

and this gives the inequality

$$
|v c(v) / d(v)|<1, \quad v \in \mathscr{V} .
$$

Defining

and

$$
\cos \gamma(\varepsilon, v)=\frac{d e(1-\cos \varepsilon)}{\left[d^{2} e^{2}(1-\cos \varepsilon)^{2}+a^{2}(d+v c)^{2} \sin ^{2} \varepsilon\right]^{1 / 2}}
$$

$$
\sin \gamma(\varepsilon, v)=\frac{a(d+v c) \sin \varepsilon}{\left[d^{2} e^{2}(1-\cos \varepsilon)^{2}+a^{2}(d+v c)^{2} \sin ^{2} \varepsilon\right]^{1 / 2}},
$$

with $\sin \gamma(0, v)=\sin \gamma(+0, v)=1$ and $\cos \gamma(0, v)=\cos \gamma(+0, v)=0$, we calculate

$$
\begin{aligned}
& X(\varepsilon, v)=C^{(1,2)}(\pi-\gamma(\varepsilon, v)) L(\varepsilon, v) C^{(1,2)}(-\gamma(\varepsilon, v)) \\
& =\left(\begin{array}{cccc}
\frac{d+v c \cdot \cos \varepsilon}{d+v c} & \varphi_{1}(a, c, d, e, v, \varepsilon) & 0 \ldots 0 & \varphi_{2}(a, c, d, e, v, \varepsilon) \\
0 & 1 & 0 \ldots 0 & 0 \\
0 & 0 & 1 \ldots 0 & 0 \\
\ldots \ldots \ldots \ldots \ldots \ldots \ldots \ldots \ldots \ldots \ldots \ldots & \ldots \ldots \ldots \ldots \ldots
\end{array} \ldots\right.
\end{aligned}
$$

where

$$
\begin{gathered}
\varphi_{1}(a, c, d, e, v, \varepsilon) \\
=\frac{\sin \varepsilon\left\{d e^{2}(1-\cos \varepsilon)\left[d^{2} e^{2}(1-\cos \varepsilon)+a^{2}(d+v c)^{2} \cos \varepsilon\right]\right.}{a e(d+v c)\left[d^{2} e^{2}(1-\cos \varepsilon)^{2}\right.} \\
\frac{\left.-a^{2}(d+v c)\left[a^{2}(d+v c)^{2} \sin ^{2} \varepsilon-d e^{2}(1-\cos \varepsilon)(d \cdot \cos \varepsilon+v c)\right]\right\}}{\left.+a^{2}(d+v c)^{2} \sin ^{2} \varepsilon\right]}, \\
\varphi_{2}(a, c, d, e, v, \varepsilon)=\frac{-v\left[d^{2} e^{2}(1-\cos \varepsilon)^{2}+a^{2}(d+v c)^{2} \sin ^{2} \varepsilon\right]^{1 / 2}}{e(d+v c)} \\
\varphi_{3}(a, c, d, e, v, \varepsilon)=\frac{c e\left[2 d(1-\cos \varepsilon)+v c \sin ^{2} \varepsilon\right]}{(d+v c)\left[d^{2} e^{2}(1-\cos \varepsilon)^{2}+a^{2}(d+v c)^{2} \sin ^{2} \varepsilon\right]^{1 / 2}}
\end{gathered}
$$


and

$\varphi_{4}(a, c, d, e, v, \varepsilon)=\frac{c \sin \varepsilon(1-\cos \varepsilon)\left[e^{2} d-a^{2}(d+v c)\right]}{a(d+v c)\left[d^{2} e^{2}(1-\cos \varepsilon)^{2}+a^{2}(d+v c)^{2} \sin ^{2} \varepsilon\right]^{1 / 2}}$.

One checks that $\Delta(X(\varepsilon, v))=1$, hence $X(\varepsilon, v)$ is an element of $\mathscr{K}$ and, by Lemma $3, X(\varepsilon, v) \in \mathscr{N}$. Therefore,

$$
X(\varepsilon, v)=N(w(\varepsilon, v)),
$$

where, by $(4.27 \mathrm{~b})$,

$$
w(\varepsilon, v)=\frac{v\left[d^{2} e^{2}(1-\cos \varepsilon)^{2}+a^{2}(d+v c)^{2} \sin ^{2} \varepsilon\right]^{1 / 2}}{e(d+v c \cos \varepsilon)} .
$$

Eqs. (4.14) and (4.15) imply

$$
\varphi_{1}(a, c, d, e, v, \varepsilon)=\varphi_{4}(a, c, d, e, v, \varepsilon)=0 .
$$

One checks that for (4.30) to be satisfied it is necessary and sufficient that

$$
d(v) e^{2}(v)=a^{2}(v)[d(v)+v c(v)] .
$$

Using (4.31) we get

$d^{2} e^{2}(1-\cos \varepsilon)^{2}+a^{2}(d+v c)^{2} \sin ^{2} \varepsilon=d e^{2}\left[2 d(1-\cos \varepsilon)+v c \sin ^{2} \varepsilon\right]$.

We have from (4.27) and (4.28), $\forall \varepsilon \in[0,2 \pi)$,

$$
\begin{gathered}
e(w(\varepsilon, v))=f(w(\varepsilon, v))=1, \\
a(w(\varepsilon, v))=d(w(\varepsilon, v))=\frac{d+v c \cos \varepsilon}{d+v c}
\end{gathered}
$$

and, using (4.32),

$$
c(w(\varepsilon, v))=\frac{c\left\{d\left[2 d(1-\cos \varepsilon)+v c \sin ^{2} \varepsilon\right]\right\}^{1 / 2}}{d(d+v c)}
$$

and

$$
w(\varepsilon, v)=\frac{v\left\{d\left[2 d(1-\cos \varepsilon)+v c \sin ^{2} \varepsilon\right]\right\}^{1 / 2}}{d+v c \cos \varepsilon} .
$$

From $(4.33 \mathrm{~b}, \mathrm{c}, \mathrm{d})$ we get, $\forall \varepsilon \in[0,2 \pi)$,

$$
\begin{gathered}
1+w^{2}(\varepsilon, v) \frac{c(v)}{v d(v)}>0 \\
a(w(\varepsilon, v))=\left(1+\frac{c(v)}{v d(v)} w^{2}(\varepsilon, v)\right)^{-1 / 2}
\end{gathered}
$$

and

$$
c(w(\varepsilon, v))=\frac{c(v)}{v d(v)} w(\varepsilon, v)\left(1+\frac{c(v)}{v d(v)} w^{2}(\varepsilon, v)\right)^{-1 / 2}
$$


From (4.25), (4.32), and (4.33d) we see that $w(\varepsilon, v)$ is a continuous function of $\varepsilon$ which equals 0 iff $\varepsilon=0$ and which, $\forall \varepsilon \in(0,2 \pi)$, has a definite sign. Then $w([0,2 \pi), v)=w([0,2 \pi], v)$ is a closed non zero interval and we write $w([0,2 \pi), v)=\left[0, w_{0}(v)\right]$ or $w([0,2 \pi), v)=\left[-w_{0}(v), 0\right]$ according to whether $w(\varepsilon, v) \geqq 0$ or $w(\varepsilon, v) \leqq 0$. In the first case, $w_{0}(v)=\operatorname{Sup}_{\varepsilon \in[0,2 \pi)} w(\varepsilon, v)$, in the second case $w_{0}(v)=-\operatorname{Inf}_{\varepsilon \in[0,2 \pi)} w(\varepsilon, v)$. From (4.33b) we get that $N^{-1}(w(\varepsilon, v))=N(-w(\varepsilon, v))$. Therefore, $-w(\varepsilon, v) \in \mathscr{V}$ and $\{N(-w(\varepsilon, v))$, $N(w(\varepsilon, v))\}_{\varepsilon \in[0,2 \pi)}=N(\Gamma(v))$ where $\Gamma(v)=\left[-w_{0}(v), w_{0}(v)\right] \subseteq \mathscr{V}, w_{0}(v)>0$. Now let $v^{\prime} \in \mathscr{V}, v^{\prime} \neq v, v^{\prime} \neq 0$. We have either $\Gamma(v) \supseteqq \Gamma\left(v^{\prime}\right)$ or $\Gamma\left(v^{\prime}\right) \supseteqq \Gamma(v)$ and $\exists \varepsilon, \varepsilon^{\prime} \in(0,2 \pi)$ such that $w(\varepsilon, v)=w\left(\varepsilon^{\prime}, v^{\prime}\right)$. Therefore $a(w(\varepsilon, v))=a\left(w\left(\varepsilon^{\prime}, v^{\prime}\right)\right)$ and we get, from (4.35),

$$
\frac{c(v)}{v d(v)}=\frac{c\left(v^{\prime}\right)}{v^{\prime} d\left(v^{\prime}\right)}=-\lambda .
$$

Thus for a given $v \in \mathscr{V}, v \neq 0$ and for $w \in \Gamma(v)$ we have, by $(4.10,33 \mathrm{a}$, $33 \mathrm{~b}, 35,36,37)$,

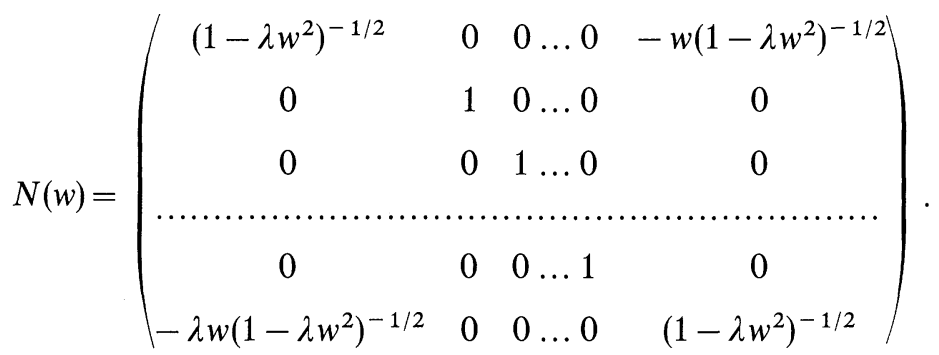

Suppose $\lambda<0$. Choose a positive integer $m$ such that $|\lambda|^{-1 / 2} \operatorname{tg}(\pi / m) \in \Gamma(v)$. One checks that

$$
\left\{\left[N\left(|\lambda|^{-1 / 2} \operatorname{tg}(\pi / m)\right)\right]^{m}\right\}_{\mu, n+1}=-\delta_{\mu, n+1}
$$

which contradicts (3.5). Therefore the circumstance $\lambda<0$ must be ruled out. We shall then distinguish two cases, according to whether $\lambda=0$ or $\lambda>0$.

If $\lambda=0,(4.38)$ is the matrix of a special Galilei transformation along the $x_{1}$-axis. Hence $N(\Gamma(v))$ generates the one-dimensional (proper orthochronous) Galilei group, which of course includes $N(v)$ because $\mathscr{V}=(-\infty,+\infty)$. Thus we get from (4.23) that $\mathscr{L}=\mathscr{G}(n)=\mathscr{G}_{0}$.

If $\lambda>0, N(\Gamma(v))$ generates the group $N\left[\left(-\lambda^{-1 / 2}, \lambda^{-1 / 2}\right)\right]$ of special (proper orthochronous) Lorentz transformations along the $x_{1}$-axis, corresponding to an invariant velocity $c=\lambda^{-1 / 2}$. From (4.37) we get $|v c(v) / d(v)|=\lambda v^{2}$, which, combined with (4.26), gives $\lambda v^{2}<1$. Hence $N\left[\left(-\lambda^{-1 / 2}, \lambda^{-1 / 2}\right)\right]=\mathscr{N}$ and using (4.23) and recalling the standard 
Wigner decomposition of the matrices of the Lorentz group [6, 7], we get that $\mathscr{L}=\mathscr{L}(n, \lambda)=\mathscr{G}_{\lambda}$. The proof of our theorem is thus completed.

Now suppose that we allow for space reflection to be included among the "rest" transformations, thus replacing condition (3.5) by

$$
\overline{\mathscr{L}} \cap \mathscr{H}(n)=\overline{\mathscr{C}}(n) .
$$

Then the proof of Theorem 1 can be adapted without essential changes to the demonstration as a corollary of the following

Theorem 2. Let $n \geqq 3$ and denote by $\bar{\Phi}$ the family of subgroups of

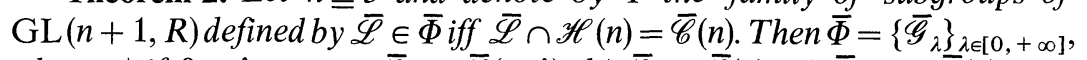
where a) if $\left.\left.0<\lambda<+\infty, \overline{\mathscr{G}}_{\lambda}=\overline{\mathscr{L}}(n, \lambda) ; b\right) \overline{\mathscr{G}}_{0}=\overline{\mathscr{G}}(n) ; c\right) \overline{\mathscr{G}}_{+\infty}=\overline{\mathscr{C}}(n)$.

\section{The Cases $n=1$ and $n=2$}

If $n=1$, conditions (3.5) and (4.39) read respectively

$$
\mathscr{L} \cap \mathscr{H}(1)=\{I\} \quad \text { and } \quad \overline{\mathscr{L}} \cap \mathscr{H}(1)=\{I, \sigma\}, \quad \sigma=\left[\begin{array}{rr}
-1 & 0 \\
0 & 1
\end{array}\right] .
$$

Both Theorem 1 and Theorem 2 do not hold any longer, in that new solutions appear. Among these, for the case without space reflection, we list the following examples:

a) the (proper orthochronous) Galilei groups respectively with rational and integer values of the velocity,

$\mathscr{G}^{(1)}=\left\{G \mid G \in \mathrm{GL}(2, R) ; G_{21}=0, G_{11}=G_{22}=1, G_{12}\right.$ integer $\}$

and

$\mathscr{G}^{(2)}=\left\{G \mid G \in \mathrm{GL}(2, R) ; G_{21}=0, G_{11}=G_{22}=1, G_{12}\right.$ rational $\}$

and

b) the Parker groups [8],

$$
\mathscr{P}_{\lambda}=\mathscr{L}(1, \lambda) \otimes\left\{\left(\begin{array}{ll}
1 & 0 \\
0 & 1
\end{array}\right),\left(\begin{array}{cc}
0 & \lambda^{-1 / 2} \\
\lambda^{1 / 2} & 0
\end{array}\right)\right\}, \quad 0<\lambda<+\infty .
$$

$\mathscr{G}^{(2)}$ is a non closed (hence non Lie) subgroup of GL $(2, R) \cdot \mathscr{G}^{(1)}$ and the $\mathscr{P}_{\lambda}$ 's are non connected Lie subgroups of $\operatorname{GL}(2, R)\left(\mathscr{G}^{(1)}\right.$ is zero-dimensional).

The groups generated by $\sigma$ with, respectively, $\mathscr{G}^{(1)}, \mathscr{G}^{(2)}$, and each of the $\mathscr{P}_{\lambda}$ 's, are solutions of the $n=1$ case with space reflection.

The fact that no kinematical groups analogous to the groups (5.3) appear for $n=3$, seems to give little relevance to the extended relativity principle formulated in Ref. [8]. 
If $n=2$, Eq. (4.13) writes $K_{12}=K_{12} D_{22}$ where $D_{22}=1$ or $D_{22}= \pm 1$ according to whether one looks for groups satisfying (3.5) or (4.39). In the second case, one can again conclude that $K_{12}=0$ (compare (4.14)) and, similarly, that $K_{32}=0$ (compare (4.15)), while this is no longer possible in the first case. Therefore, for $n=2$, the proof which we have given in Section 4 is still valid for Theorem 2, but fails for Theorem $1^{3}$.

We have not been able to ascertain whether Theorem 1 extends or not to the case $n=2^{4}$. However, we can prove a weaker result which is given by the following

Proposition. Denote by $\Psi$ the family of subgroups of $\mathrm{GL}(3, R)$ defined by $\mathscr{M} \in \Psi$ iff $\mathscr{M}$ is a connected Lie subgroup of $\mathrm{GL}(3, R)$ satisfying

$$
\mathscr{M} \cap \mathscr{H}(2)=\mathscr{C}(2) \text {. }
$$

Then $\Psi=\left\{\mathscr{M}_{\lambda}\right\}_{\lambda \in[0,+\infty]}$, where a) if $0<\lambda<+\infty, \mathscr{M}_{\lambda}=\mathscr{L}(2, \lambda)$; b) $\mathscr{M}_{0}=\mathscr{G}(2)$; c) $\mathscr{M}_{+\infty}=\mathscr{C}(2)$.

Proof. Consider the following subalgebras of $\mathrm{gl}(3, R)$, the Lie algebra of all $3 \times 3$ real matrices:

and

$$
h(3, R)=\left\{h \mid h \in \operatorname{gl}(3, R) ; h_{13}=h_{23}=0\right\}
$$

$$
s o(2, R)=\left\{s \mid s \in \operatorname{gl}(3, R) ; s=\alpha m_{12} ; \alpha \text { real; } m_{12}=e_{12}-e_{21}\right\},
$$

where $\left\{e_{\varrho \sigma}\right\}_{\varrho, \sigma=1,2,3}$ is the basis for $\operatorname{gl}(3, R)$ given by $\left(e_{\varrho \sigma}\right)_{\lambda \nu}=\delta_{\varrho \lambda} \delta_{\sigma \nu}$. $\mathrm{gl}(3, R), h(3, R)$ and $s o(2, R)$ can be identified, respectively, to the Lie algebras of $\mathrm{GL}(3, R), \mathscr{H}(2)$ and $\mathscr{C}(2)$. If $\mathscr{M}$ is a Lie subgroup of $\mathrm{GL}(3, R)$ satisfying (5.4) and if we denote by $\Lambda(\mathscr{M})$ its Lie algebra identified canonically to a subalgebra of $\mathrm{gl}(3, R)$, then

$$
\Lambda(\mathscr{M}) \cap h(3, R)=\operatorname{so}(2, R) .
$$

Therefore, we look for those subalgebras $\Lambda$ of $g l(3, R)$ which satisfy

$$
\Lambda \cap h(3, R)=\operatorname{so}(2, R) .
$$

Let $\Lambda$ satisfy (5.8). Then $\operatorname{dim} \Lambda \leqq 3$. If $\operatorname{dim} \Lambda=1$, then

$$
\Lambda=\Lambda_{+\infty}=\operatorname{so}(2, R) .
$$

Suppose $\operatorname{dim} \Lambda=2$ and let $\left\{m_{12}, p\right\}$ be a basis for $\Lambda$. We write

$$
p=\sum_{\varrho, \sigma=1}^{3} \mu_{\varrho \sigma} e_{\varrho \sigma}
$$

${ }^{3}$ This failure is due to the fact that if space is two-dimensional one has no freedom to rotate around a space axis. In the cases $n \geqq 3$, this freedom implies through (4.14) and (4.15) that normality to the direction of motion is a frame independent property.

${ }^{4}$ If not, it would result that the dimension of physical space is the lowest for which only the Lorentz and Galilei groups appear as linear kinematical groups compatible with the isotropy of space. 
and we have

$$
\begin{aligned}
{\left[m_{12}, p\right] } & =\left(\mu_{12}+\mu_{21}\right)\left(e_{11}-e_{22}\right)+\left(\mu_{22}-\mu_{11}\right)\left(e_{12}+e_{21}\right) \\
& +\mu_{23} e_{13}-\mu_{13} e_{23}+\mu_{32} e_{31}-\mu_{31} e_{32} .
\end{aligned}
$$

(5.10) shows that $\Lambda$ abelian would imply $\mu_{13}=\mu_{23}=0$, which contradicts (5.8). If $\Lambda$ is non abelian we can choose $p$ in such a way that $\left[m_{12}, p\right]$ $=\beta \mathrm{p}, \beta \neq 0$.

Then (5.10) writes

$$
\begin{aligned}
& \beta\left[\mu_{11}\left(e_{11}-e_{22}\right)+\mu_{12}\left(e_{12}+e_{21}\right)+\mu_{13} e_{13}+\mu_{23} e_{23}+\mu_{31} e_{31}+\mu_{32} e_{32}\right] \\
& =2 \mu_{12}\left(e_{11}-e_{22}\right)-2 \mu_{11}\left(e_{12}+e_{21}\right)+\mu_{23} e_{13}-\mu_{13} e_{23}+\mu_{32} e_{31}-\mu_{31} e_{32}
\end{aligned}
$$

and inspection shows that this implies $p=0$. Hence there are no twodimensional subalgebras of $\mathrm{gl}(3, R)$ satisfying (5.8). Now suppose $\operatorname{dim} \Lambda=3$ and let $\left\{m_{12}, p, q\right\}$ be a basis for $\Lambda$. Write

$$
p=\sum_{\varrho, \sigma=1}^{3} \mu_{\varrho \sigma} e_{\varrho \sigma}, \quad q=\sum_{\varrho, \sigma=1}^{3} v_{\varrho \sigma} e_{\varrho \sigma}
$$

and the brackets

$$
\begin{aligned}
{\left[m_{12}, p\right] } & =\alpha m_{12}+\beta p+\gamma q, \\
{\left[m_{12}, q\right] } & =\alpha^{\prime} m_{12}+\beta^{\prime} p+\gamma^{\prime} q, \\
{[p, q] } & =\alpha^{\prime \prime} m_{12}+\beta^{\prime \prime} p+\gamma^{\prime \prime} q .
\end{aligned}
$$

$\Lambda$ cannot contain a two-dimensional subalgebra to which $m_{12}$ belongs. Therefore we can confine ourselves to the consideration of the following cases: a) $\beta \neq 0, \gamma \neq 0, \beta^{\prime} \neq 0, \gamma^{\prime} \neq 0$, b) $\beta \neq 0, \gamma \neq 0, \beta^{\prime} \neq 0, \gamma^{\prime}=0$, c) $\beta=0$, $\gamma \neq 0, \beta^{\prime} \neq 0, \gamma^{\prime} \neq 0$ and d) $\beta=\gamma^{\prime}=0, \gamma \neq 0, \beta^{\prime} \neq 0$.

Cases b) and c) are equivalent because they can be reduced one to the other by the exchange $p \rightleftarrows q$. As to case a), one checks that it can be reduced to case b) by a suitable change of basis in the subspace $\{p, q\}$. Consider case b). Inserting (5.11) into (5.12a, b) we get, in particular,

$$
\begin{aligned}
\left(\gamma+\beta^{\prime-1}\right) v_{13}+\beta v_{23} & =0, \\
-\beta v_{13}+\left(\gamma+\beta^{\prime-1}\right) v_{23} & =0 .
\end{aligned}
$$

By (5.8), $v_{13}$ and $v_{23}$ cannot both vanish, so it must be $\beta=0$ and $\beta^{\prime}=-\gamma^{-1}$. Therefore case b) reduces to case d) for which, with the replacements $p \rightarrow-\gamma \alpha^{\prime} m_{12}+p$ and $q \rightarrow \alpha m_{12}+\gamma q$, the brackets write

$$
\begin{aligned}
{\left[m_{12}, p\right] } & =q, \\
{\left[m_{12}, q\right] } & =-p, \\
{[p, q] } & =\delta m_{12}+\zeta p+\eta q .
\end{aligned}
$$


Substituting (5.11) into $(5.13 \mathrm{a}, \mathrm{b})$ we get

and

$$
\mu_{11}=\mu_{22}=\mu_{33}=\mu_{21}=\mu_{12}=v_{11}=v_{22}=v_{33}=v_{21}=v_{12}=0
$$

$$
v_{13}=\mu_{23}, \quad v_{23}=-\mu_{13}, \quad v_{31}=\mu_{32}, \quad v_{32}=-\mu_{31},
$$

whence

and

$$
p=\mu_{13} e_{13}+\mu_{23} e_{23}+\mu_{31} e_{31}+\mu_{32} e_{32}
$$

$$
q=\mu_{23} e_{13}-\mu_{13} e_{23}+\mu_{32} e_{31}-\mu_{31} e_{32} .
$$

Substitution into $(5.13 \mathrm{c})$ gives

and

$$
[p, q]=-\left(\mu_{13} \mu_{31}+\mu_{32} \mu_{23}\right) m_{12}
$$

$$
\mu_{32} \mu_{13}=\mu_{31} \mu_{23} .
$$

Because of (5.8), $\mu_{13}$ and $\mu_{23}$ cannot both vanish and we can confine ourselves to the consideration of the case $\mu_{23} \neq 0$ (by possibly performing the replacements $p \rightarrow q$ and $q \rightarrow-p$ ). Then, setting $\mu_{13} / \mu_{23}=\beta, \mu_{32} / \mu_{23}=\lambda$ and performing the replacements

and

$$
p \rightarrow p(\lambda)=\left[\left(1+\beta^{2}\right) \mu_{23}\right]^{-1}(p-\beta q)=e_{23}+\lambda e_{32}
$$

$$
q \rightarrow q(\lambda)=\left[\left(1+\beta^{2}\right) \mu_{23}\right]^{-1}(\beta p+q)=e_{13}+\lambda e_{31},
$$

we get the new brackets

$$
\begin{aligned}
& {\left[m_{12}, p(\lambda)\right]=q(\lambda),} \\
& {\left[m_{12}, q(\lambda)\right]=-p(\lambda),} \\
& {[p(\lambda), q(\lambda)]=-\lambda m_{12} .}
\end{aligned}
$$

$\forall \lambda \in(-\infty,+\infty)$ we have a Lie algebra $\Lambda_{\lambda}$ satisfying (5.8). Thus the family $\Omega$ of subalgebras of $\mathrm{gl}(3, R)$ satisfying (5.8) can be indexed as $\Omega=\left\{\Lambda_{\lambda}\right\}_{\lambda \in(-\infty,+\infty]}$ where $\Lambda_{+\infty}$ is given by (5.9) and, if $-\infty<\lambda<+\infty$, $\Lambda_{\lambda}$ has a basis $\left\{m_{12}, p(\lambda), q(\lambda)\right\}$, where $m_{12}=e_{12}-e_{21}$ and $p(\lambda)$ and $q(\lambda)$ are given by (5.18) and (5.19) respectively. $\forall \lambda \in(-\infty,+\infty]$ denote by $\mathscr{G}\left(\Lambda_{\lambda}\right)$ the (unique) connected Lie subgroup of GL $(3, R)$ of which $\Lambda_{\lambda}$ is the Lie algebra. Then $\mathscr{G}\left(\Lambda_{+\infty}\right)=\mathscr{C}(2), \mathscr{G}\left(\Lambda_{0}\right)=\mathscr{G}(2)$ and, if $0<\lambda<+\infty$, $\mathscr{G}\left(\Lambda_{\lambda}\right)=\mathscr{L}(2, \lambda)$. These groups all satisfy (5.4). On the other hand, if $-\infty<\lambda<0$,

$$
\begin{aligned}
& \mathscr{G}\left(\Lambda_{\lambda}\right)=\left\{L \mid L \in \mathrm{GL}(3, R) ; L^{T} g(\lambda) L=g(\lambda) ;\right. \\
& \left.g_{\mu \nu}(\lambda)=0 \text { if } \mu \neq v ; g_{11}(\lambda)=g_{22}(\lambda)=|\lambda| ; g_{33}(\lambda)=1 ; \operatorname{det} L=1\right\},
\end{aligned}
$$


and these groups do not satisfy (5.7) because they all contain the matrix $\operatorname{diag}\{-1,1,-1\}$. The proposition is thus proved.

Acknowledgements. The author is indebted to Professor H. D. Doebner for fruitful discussions. Herewith he also acknowledges gratefully a fellowship from the Alexander von Humboldt-Stiftung, Bad Godesberg.

\section{References}

1. Lalan, V.: Bull. Soc. Math. France 65, 83 (1937).

2. Bacry, H., Lévy-Leblond, J.-M.: J. Math. Phys. 9, 1605 (1968).

3. Gorini, V.: Derivation of the Lorentz and Galilei groups from rotational invariance. Seminar delivered at the International Advanced Study Institute on Mathematical Physics, Robert College, Istanbul, Turkey, August 10-21, 1970. To appear in the proceedings.

4. Berzi,V., Gorini, V.: Space-time, reference frames, relativistic invariance: a topological approach. Milan University report IFUM 108/FT-1970. Unpublished.

5. Gantmacher,F.R.: The theory of matrices, Vol. 1, Chapter IX, p. 285. New York: Chelsea Publ. Comp. 1959.

6. Wigner, E. P.: Ann. Math. 40, 149 (1939).

7. Takahashi, R.: Bull. Soc. Math. France 91, 306 (1963).

8. Parker, L.: Phys. Rev. 188, 2287 (1969).

\section{Gorini}

Institut für Theoretische Physik (I)

der Universität Marburg

BRD-3550 Marburg (Lahn). Germany 